\title{
Preimages for Reduced SHA-0 and SHA-1
}

\author{
Christophe De Cannière ${ }^{1,2}$ and Christian Rechberger ${ }^{3}$ \\ ${ }^{1}$ Département d'Informatique École Normale Supérieure \\ christophe.decanniere@ens.fr \\ ${ }^{2}$ Katholieke Universiteit Leuven, Dept. ESAT/SCD-COSIC, and IBBT \\ ${ }^{3}$ Graz University of Technology \\ Institute for Applied Information Processing and Communications (IAIK) \\ christian.rechberger@iaik.tugraz.at
}

\begin{abstract}
In this paper, we examine the resistance of the popular hash function SHA-1 and its predecessor SHA-0 against dedicated preimage attacks. In order to assess the security margin of these hash functions against these attacks, two new cryptanalytic techniques are developed:
\end{abstract}

- Reversing the inversion problem: the idea is to start with an impossible expanded message that would lead to the required digest, and then to correct this message until it becomes valid without destroying the preimage property.

- $\mathbf{P}^{3}$ graphs: an algorithm based on the theory of random graphs that allows the conversion of preimage attacks on the compression function to attacks on the hash function with less effort than traditional meet-in-the-middle approaches.

Combining these techniques, we obtain preimage-style shortcuts attacks for up to 45 steps of SHA-1, and up to 50 steps of SHA-0 (out of 80).

Keywords: hash function, cryptanalysis, preimages, SHA-0, SHA-1, directed random graph.

\section{Introduction}

Until recently, most of the cryptanalytic research on popular dedicated hash functions has focused on collisions resistance, as can be seen from the successful attempts to violate the collision resistance property of MD4 [10], MD5 [32, 34], SHA-0 6] and SHA-1 [13, 21, 33] using the basic ideas of differential cryptanalysis [2]. The community developed a wealth of fairly sophisticated tools that aid this type of analysis, including manual [33] and automated [7, 8, 20] methods to search and evaluate characteristics optimized for differential cryptanalysis of the used building blocks.

This wealth of results stands in stark contrast to what is known about the preimage and second preimage resistance of these hash functions. This is especially unsatisfying since most applications of hash functions actually rely more on preimage and second preimage resistance than on collision resistance.

Some of the Main Features of Our Results: All currently known generic preimage attacks require either impractically long first preimages [15], a first 
preimage lying in a very small subset of the set of all possible preimages [35], or a target digest constructed in a very special way [14.

In this work, we study the resistance of SHA-0 and SHA-1 against dedicated cryptanalytic attacks in settings where only relatively short preimages are allowed and a first preimage might not be available. An example of a very common use case of hash functions that relies on the resistance against these kind of attacks: hashed passwords. Especially SHA-1 is ubiquitously used, and will continue to be recommended by NIST even after 2010 outside the application of digital signatures 24, e.g., as RNG or KDF.

We exploit weak diffusion properties in the step transformation and in the message expansion to divide the effort to find a preimage, and consider only one or a small number of bits at a given time. In particular we present two new cryptanalytic tools. Firstly a compression function attack by means of correcting invalid messages, described in Sect. 3. Secondly, an algorithm based on the theory of random graphs that allows an efficient conversion of preimage attacks on the compression function to attacks on the hash function is presented in Sect. 4 .

Later, in Sect. 5 we will discuss the results of combining these methods. This results in cryptanalytic shortcuts attacks for up to 50 step of SHA-0 (out of 80) and 45 steps of SHA-1. As a proof-of-concept we give a preimage for the 33-step SHA-0 compression function and also a second preimage of an ASCII text under the SHA-0 hash function reduced to 31 steps in Appendix B.

\section{The SHA Family}

In this paper, we will focus on the hash function SHA-1 and its predecessor SHA-0. The SHA-1 algorithm, designed by the US National Security Agency (NSA) and adopted as a standard in 1995, is widely used, and is representative for a large class of hash functions which started with MD4 and includes most algorithms in use today. In this section, we only briefly review a few features of the SHA design which are important for the techniques presented in this paper. For a complete description we refer to the specifications [25].

SHA-0 and SHA-1 consist of the iterative application of a compression function (denoted by $f$ in Fig. 1), which transforms a 160-bit chaining variable $h_{j-1}$ into $h_{j}$, based on a 512-bit message block $m_{j}$. At the core of the compression function lies a block cipher $g$ which is used in Davies-Meyer mode (see Fig. 2).

The block cipher itself consists of two parts: a message expansion and a state update transformation.

The purpose of the message expansion is to expand a single 512-bit input message block into eighty 32 -bit words $W_{0}, \ldots, W_{79}$. This is done by splitting the message block into sixteen 32 -bit words $M_{0}, \ldots, M_{15}$, which are then expanded linearly according to the following recursive rule:

$$
W_{i}= \begin{cases}M_{i} & \text { for } 0 \leq i<16, \\ \left(W_{i-3} \oplus W_{i-8} \oplus W_{i-14} \oplus W_{i-16}\right) \lll s & \text { for } 16 \leq i<80 .\end{cases}
$$




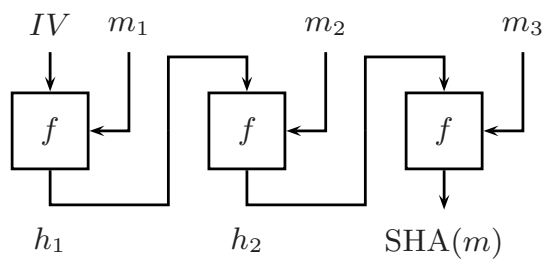

Fig. 1. An iterated hash function
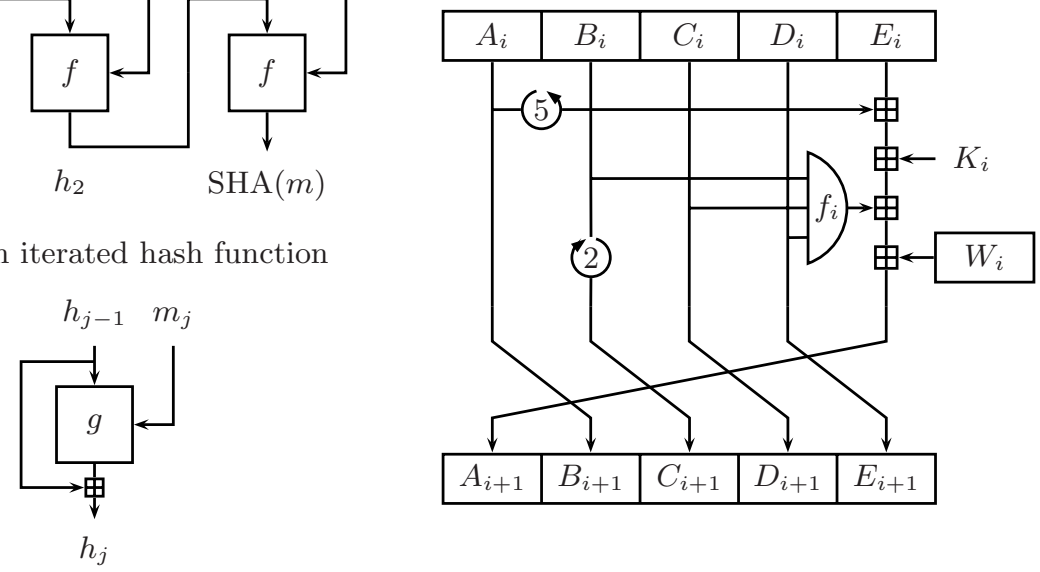

Fig. 2. The Davies-Meyer mode

Fig. 3. A single state update step

The only difference between SHA-0 and SHA-1 lies in the rotation value $s$, which is 0 for SHA-0, and 1 for SHA-1.

The state update transformation takes as input a 160-bit chaining variable $h_{j-1}$ which is used to initialize five 32 -bit registers $A, B, \ldots, E$. These registers, referred to as state variables, are then iteratively updated in 80 steps, one of which is shown in Fig. 3. Note that the state update transformation can also be described recursively in terms of $A_{i}$ only: after introducing $A_{-1}=B_{0}, A_{-2}=$ $C_{0} \lll 2, A_{-3}=D_{0} \lll 2$, and $A_{-4}=E_{0} \lll 2$, we can write:

$$
A_{i+1}=\left(A_{i} \lll 5\right)+W_{i}+f\left(A_{i-1}, A_{i-2} \ggg 2, A_{i-3} \ggg 2\right)+\left(A_{i-4} \ggg 2\right)+K_{i} .
$$

Because of this property, we will only consider the state variable $A_{i}$ in the remainder of this paper.

\section{Inverting the Compression Function}

Before devising (second-) preimage attacks against the complete SHA function, we first focus on its compression function, and develop inverting methods which will be used as building blocks afterwards.

\subsection{Possible Approaches}

The recent successes in constructing collisions in SHA-0 and SHA-1 raise the natural question whether the differential techniques developed for collision attacks could also be used for constructing preimages. The question is especially pertinent in the case of second preimages, which are in fact just special types of collisions. 
A first straightforward approach would consist in reusing the differential characteristics used in collision attacks by applying the corresponding message difference to the given message. If the characteristic is followed, then this will yield a second preimage. While this approach was applied to MD4 by Yu et al. 35], and to SHA-1 reduced to 53 steps by Rechberger and Rijmen [29, 30], it has some serious limitations when trying to find second preimages of reasonably short messages. The main problem is that, since the starting message is already fixed, the probability of the characteristic directly translates into the success probability of the attack (instead of determining the number of trials, as in collision attacks). This probability is further reduced by the fact that we lose the possibility to influence the difference propagation by fixing bits of the message to special values. In the case of MD4 and 53-step SHA-1, this results in attacks which only succeed with a probability of $2^{-56}$ and $2^{-151.5}$ respectively.

A second approach, which was recently proposed by Leurent in [17], relies on the existence of special messages which can simultaneously be combined with a large number of different characteristics, resulting in a large set of related messages. The idea is to compute the hash value of such a special message, and then apply the appropriate differences in order to steer this value towards the target value. Similar strategies have previously been used in practical second preimage attacks on SMASH by Lamberger et al. [16], and more recently in preimage attacks on GOST by Mendel et al. [18, 19]. In the case of MD4, this approach does not require a first preimage to start with, and results in a preimage attack against full MD4 with a complexity of $2^{100}$.

It is not clear, however, how these ideas could efficiently be applied to hash functions such as SHA-0 or SHA-1, which, while still being vulnerable, show much more resistance to differential cryptanalysis than MD4. In the next sections, we will therefore study a completely different approach, which, as will be seen, has little in common with the techniques used in collision attacks.

\subsection{Turning the Function Around}

The problem we are trying to solve in this section is the following: given a 160-bit target value $h_{1}$, and a 160-bit chaining input $h_{0}$, find a 512-bit message input $m_{0}$ such that $f\left(h_{0}, m_{0}\right)=h_{1}$, or equivalently that $g\left(h_{0}, m_{0}\right)=h_{1}-h_{0}$. Since the size of the message is much larger than the size of the output, we expect this equation to have a very large number of solutions. The difficulty in determining the 512 unknown input bits, however, lies in the fact that each of the 160 bitconditions imposed at the output, depends in a complicated way on all 512 input bits.

The main observation on which the inversion method proposed in this paper is based, is that we can obtain a larger, but considerably less interconnected system of equations by expressing the problem in terms of internal state variables, rather than in terms of message words. That is, instead of trying to tweak a message in the hope to be able to control its effect on the output after being expanded and fed through several iterations of the state update transformation, we will start from state variables which already produce the correct output, and modify them 


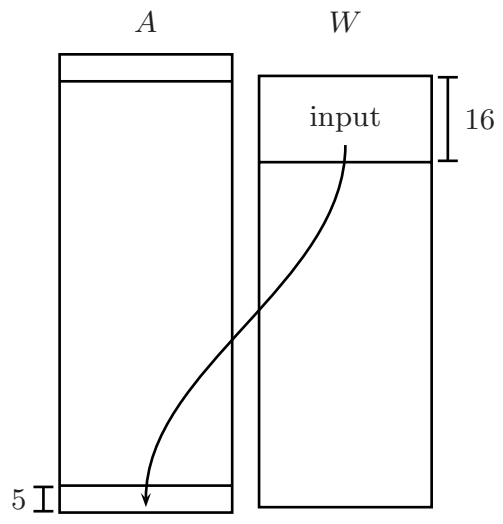

output

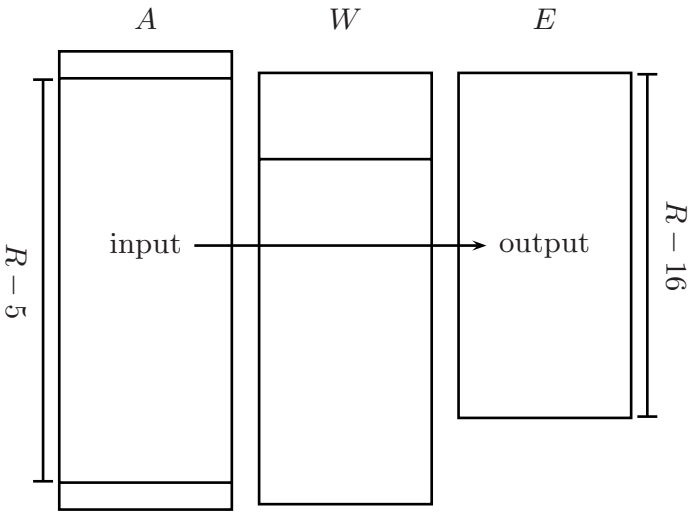

(b)

Fig. 4. Two equivalent descriptions of the inversion problem for a compression function reduced to $R$ rounds

in such a way that the expanded message words, which can easily be derived from them, satisfy the linear recursion of the message expansion.

The idea is illustrated in Fig. 4. Instead of considering the function which maps $M_{0} \ldots M_{15}$ to $A_{76} \ldots A_{80}$ as in Fig. 4(a), we will first fix $A_{76} \ldots A_{80}$ to the target value determined by $h_{1}-h_{0}$, and then analyze the function in Fig. 4(b) which maps $A_{1} \ldots A_{75}$ to error words $E_{0} \ldots E_{64}$, where

$$
\begin{aligned}
E_{i} & =W_{i} \oplus W_{i+2} \oplus W_{i+8} \oplus W_{i+13} \oplus\left(W_{i+16} \ggg s\right), \quad \text { and } \\
W_{i} & =A_{i+1}-\left(A_{i} \lll 5\right)-f\left(A_{i-1}, A_{i-2} \ggg 2, A_{i-3} \ggg 2\right)-\left(A_{i-4} \ggg 2\right)-K_{i} .
\end{aligned}
$$

Clearly, finding an input which maps to $h_{1}-h_{0}$ in Fig. 4(a) is equivalent to the problem of finding an input which maps to zero in Fig. 4(b).

The potential advantages of this alternative approach are clearly seen when analyzing how flipping a single bit in the input affects the output in both cases. In the first case, illustrated in Fig. 5(a), a single flip in the message quickly propagates through both the expanded message and the state, resulting in a completely uncontrollable pattern of changes at the output. In the second case, however, a bit-flip in the state propagates to the output in a very predictable way, as shown in Fig. 5(b). A change in the state affects at most 6 consecutive expanded messages words, and at most 22 words of the output. More importantly, depending on the position of the flipped bit in the state word, it will leave the least significant bits of all $W_{i}$ and $E_{i}$ untouched. The downside is that both the input and the output of the function to invert are considerably larger.

\subsection{Fixing Problems Column by Column}

Let us now analyze in a little bit more detail how state bits affect the output words in our new function. In order to simplify the analysis, we will for now assume that we deal with a variant of SHA-0 reduced to $R$ rounds. 


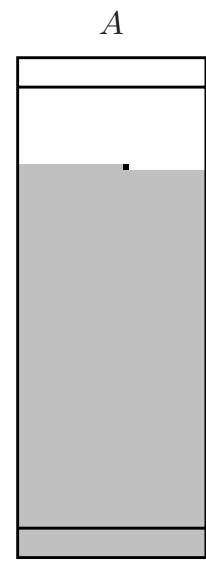

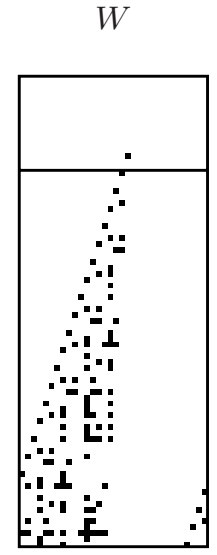

(a)
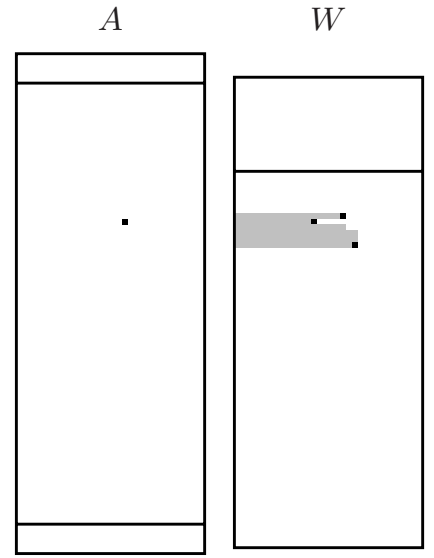

(b)

Fig. 5. Bits affected by a single bit flip at the input (SHA-1). Black bits are guaranteed to flip; gray bits may be flipped; white bits are unaffected.

Suppose that we restrict ourselves to the first $j+1$ bits of each expanded message word $W_{i}$ (denoted by $W_{i}^{j \cdots 0}$ ), and that we keep all state bits constant except for those at bit position $j+2$ (referred to as $a_{i}^{j+2}$ ). In this case, we can derive a simple relation (by collecting all constant parts into a $j+1$-bit word $C_{i}^{j \cdots 0}$ and a 1-bit variable $c_{i}^{j}$ ), which holds as long as $0 \leq j<25$ :

$$
W_{i}^{j \cdots 0}=C_{i}^{j \cdots 0}-\left(f\left(c_{i}^{j}, a_{i-2}^{j+2}, a_{i-3}^{j+2}\right) \ll j\right)-\left(a_{i-4}^{j+2} \ll j\right) .
$$

The interesting property of this relation is that the effect of the state bits $a_{i}^{j+2}$ is confined to the most significant bit of $W_{i}^{j \cdots 0}$. Furthermore, this effect is linear in all rounds where $f_{\mathrm{XOR}}$ or $f_{\mathrm{IF}}$ is used. Since the words $E_{i}$ in SHA-0 are just a bitwise XOR of expanded message words $W_{i}$, this property holds for those words as well.

We can now use this observation to gradually fix the bits of $E_{i}$ to zero, column by column. We start by determining $a_{1}^{2} \ldots a_{R-5}^{2}$ such that the least significant bits of all $R-16$ output words $E_{i}$ are zero. Since we have $R-5$ degrees of freedom and only need to satisfy $R-16$ conditions, we expect to find $2^{11}$ different solutions. Thanks to the special structure of the equations, these solutions can be found recursively with a computation effort which is linear in the number of rounds $R$. Next, we use $a_{1}^{3} \ldots a_{R-5}^{3}$ (which, as indicated by (11), will not affect the least significant bits) to correct the second least significant bits. We proceed this way as long as (1) holds, and eventually we will only be left with non-zero bits in the 7 most significant bits of the $R-16$ output words.

In order to eliminate the remaining non-zero bits, we could just repeat the previous procedure with different solutions for the state bits, until these non-zero bits disappear by themselves. This would require in the order of $2^{7 \cdot(R-16)}$ trials. In the next section, we will show how this number can be reduced. 
A

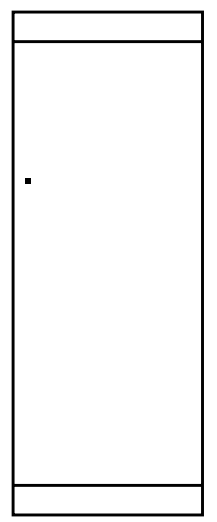

W

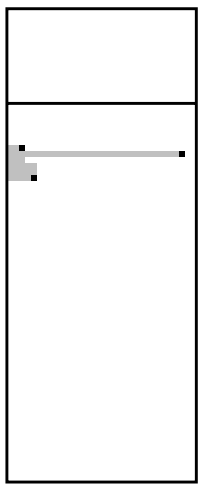

(a)
E

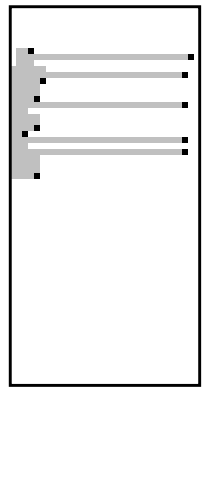

W

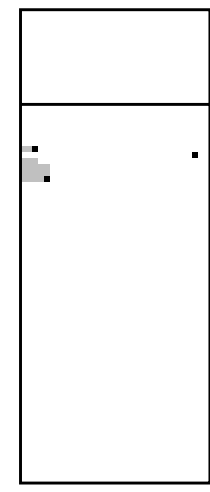

(b)

Fig. 6. Flipping state bit 29 with (a) and without (b) carries (SHA-1)

\subsection{Preventing Carries}

A natural way to improve the previous attack is to try to extent the property found in (11) to the case $j \geq 25$. The problem however is that the equation gets an extra term for $25 \leq j<30$ :

$$
W_{i}^{j \cdots 0}=C_{i}^{j \cdots 0}-\left(a_{i}^{j+2} \ll j-25\right)-\left(f\left(c_{i}^{j}, a_{i-2}^{j+2}, a_{i-3}^{j+2}\right) \ll j\right)-\left(a_{i-4}^{j+2} \ll j\right) .
$$

Hence, when trying to fix the output bits in column $j$, we have to make sure that this extra term at position $j-25$ does not reintroduce errors in the previously fixed columns. In order to do so, we will first try to confine the potential trouble caused by this term to a single column by preventing the propagation of carries to other columns (the idea is shown in Fig. 6). This can easily be achieved by noting that the 5 most significant bits of $A_{i}$, which we are currently trying to determine, affect the least significant part of $W_{i}$ through the equation $W_{i}=X_{i}-\left(A_{i} \lll 5\right)$, where

$$
X_{i}=A_{i+1}-f\left(A_{i-1}, A_{i-2} \ggg 2, A_{i-3} \ggg 2\right)-\left(A_{i-4} \ggg 2\right)-K_{i} .
$$

If we now choose the 7 least significant columns of the state in beforehand in such a way that there are no zeros in the 5 least significant bits of $X_{i}$, then no carries (borrows) will appear later on when the 5 most significant bits of $A_{i}$ are modified. Once these 7 columns have been determined, we start correcting the output columns for $5 \leq j<25$ in exactly the same way as explained in the previous section.

When we arrive at $j \geq 25$, we will try to use the state bits at position $j+2$ to simultaneously correct columns $j$ and $j-25$ of the output. This time, we have $R-5$ degrees of freedom to satisfy $2 \times(R-16)$ conditions, and hence we will still have to rely on chance for $R-27$ of these conditions. In total, we will 
leave $5 \times(R-27)$ uncorrected output bits in columns $25-29$ and $2 \times(R-16)$ in columns 30-31. As a consequence, we will need to perform $2^{c}$ trials with $c=2 \cdot(R-16)+5 \cdot(R-27)$ in order for all non-zero bits to be eliminated.

\subsection{Relaxing the Problem: Partial-Pseudo-Preimages}

In the previous section, we had to leave a number of output bits uncorrected because of a lack of degrees of freedom in the state bits in columns 27-31. One way to create up to 10 additional degrees of freedom in each of these 5 columns is to allow the attacker to modify bits $a_{-4}^{j} \ldots a_{0}^{j}$ and/or $a_{R-4}^{j} \ldots a_{R}^{j}$ as well. In this case, the input and the output of the compression function will only partially match $h_{0}$ and $h_{1}$, and we call this a partial-pseudo-preimage. It is easy to see that each additional degree of freedom will reduce the cost by a factor two, i.e., if we allow $b_{1} \leq 25$ input bits and $b_{2} \leq 25$ output bits to deviate from their original target, then the computation effort of finding a partial-pseudo-preimage will be given by

$$
2^{c}, \quad \text { where } c=2 \cdot(R-16)+5 \cdot(R-27)-\left(b_{1}+b_{2}\right) .
$$

\subsection{Application to SHA-1}

The techniques explained for SHA-0 can be applied to SHA-1 in a relatively straightforward way. The only difference is that affected bits in $W_{i}$, with $i \geq 16$, will not only propagate to the corresponding columns in the error words, but also to the columns shifted by one position to the right. In order to compensate for this, it suffices to consider different state bits when correcting the columns, i.e., instead of using $a_{1}^{j+2} \ldots a_{R-5}^{j+2}$ to correct column $j$ (and $j-25$ if $j \geq 25$ ), we will now use the state bits $a_{1}^{j+2} \ldots a_{11}^{j+2}$ and $a_{12}^{j+3} \ldots a_{R-5}^{j+3}$. This works fine as long as $j<29$. The bits $a_{12}^{j+3} \ldots a_{R-5}^{j+3}$ cannot be used anymore when $j=29$, though. Since we lose $R-16$ degrees of freedom for fixing the last pair of columns (columns 29 and 4 ), the computational effort increases to:

$$
2^{c}, \quad \text { where } c=3 \cdot(R-16)+5 \cdot(R-27)-\left(b_{1}+b_{2}\right) .
$$

In addition to this, and for the same reason, we can now only fully exploit 20 additional degrees of freedom at the output, i.e., $b_{2} \leq 20$. We still have $b_{1} \leq 25$, though.

\section{Preimages from Partial-Pseudo-Preimages $-\mathbf{P}^{3}$ graphs}

For the discussion in this section, let's assume we are given a method to produce partial-pseudo-preimages that is faster than a method to find preimages directly.

We first discuss a number of well understood methods in Sect. 4.1 that transform such attacks on the compression function into a preimage attack on the hash function by means of meet-in-the-middle and tree building techniques. Next, in Sect. 4.2 we discuss a new method using so-called $\mathrm{P}^{3}$ graphs, that makes it possible to exploit the existence of such weaker attacks more directly. 
(a)

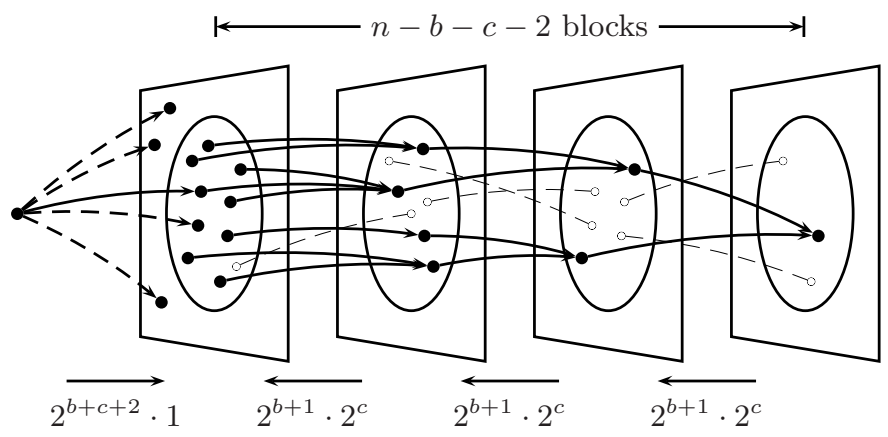

(b)

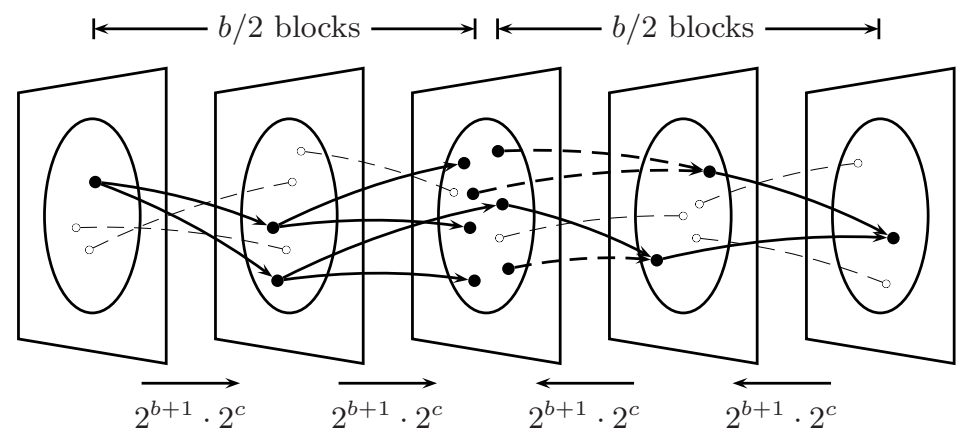

(c)

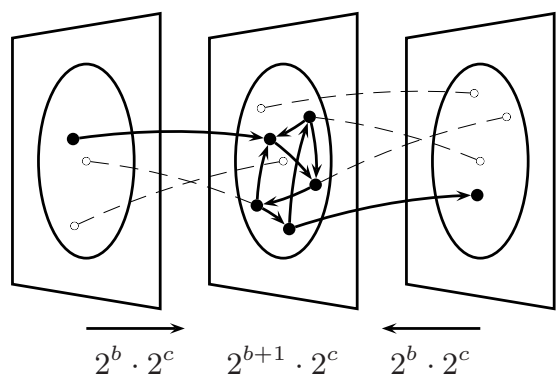

Fig. 7. Three different ways to build preimages from partial pseudo-preimages

\subsection{Meet-in-the-Middle and Tree Based Methods}

Inverting a Davis-Meyer compression function is the problem of finding a pair $(h, m)$ such that $g(h, m)+h$ equals a given digest d. It was shown that no black-box attack can give a preimage faster than essentially $2^{n}[3,27]$. Inverting a Merkle-Damgård hash function is the problem of, given an initial chaining input $h_{0}$, finding an (almost arbitrarily large) number $x$ of message blocks $m_{0} \ldots m_{x}$ such that $h_{x}$ equals a given digest $d$. 
In the following, we assume that a part of the chaining input (say $n-b_{1}$ out of the $n$ bits) can be chosen by the attacker, or in other words: the attacker can control all but $b_{1}$ bits of the chaining input (always the same $n-b_{1}$ bits). Let's further assume that a partial preimage attack on the compression function (of $\operatorname{cost} 2^{c}$ ) has the property that a preimage can be found where all but $b_{2}$ out of $n$ bits match the targeted digest $d$ (again always the same $n-b_{2}$ bits). In addition to the parameters $b_{1}$ and $b_{2}$ introduced in Sect. 3.5. we will denote the number of bit positions of the chaining variable which can be controlled both from the input and from the output by $n-b$. All the following methods yield a preimage of the hash function for any given digest $d$

- Meet-in-the-middle approach 1. A basic unbalanced meet-in-the-middle approach that does not take advantage of the $b$ bits that overlap has runtime $2^{(n+c) / 2+1}$ and memory costs of $2^{(n-c) / 2}$. The balanced case appeared already in [9], memoryless variants appear to have been first proposed in [23, 28].

- Meet-in-the-middle approach 2. By using the fact that both in forwards, and backwards direction, only $b$ bits need to meet, the runtime requirement improves to $2^{b / 2+c_{1}}+2^{b / 2+c_{2}}$, where $c_{1}$ denotes the cost of a partial-preimage attack (the forward part, if no compression function attack is available, a brute force attack with this property has cost $2^{n-b}$ ), and $c_{2}$ denotes the cost of the pseudo-preimage attack (this is equivalent to calling the partialpseudo-preimage attack $2^{b}$ times at the cost of $2^{b+c}$ ). The total runtime is hence $2^{3 b / 2+c+1}$, the memory requirement is $2^{b}$.

- Layered Tree method due to Leurent, see Fig. 7(a). In [17] the following tree method was proposed. Starting from the target hash d, produce two different pseudo preimages with cost $2^{b+c+1}$. As a next step, produce four different pseudo preimages with the same cost that target both new target chaining values. This process is continued for $n-b-c-2$ blocks and needs about $2^{n-b-c-1}$ of storage. For a fixed length preimage, only the last layer of the tree can be used for random trials in the forward direction, amounting to $2^{b+c+2}$ trials. Variants with a different branch number, or with less restrictions on the way the tree grows are thinkable [17].

- Alternative Backward-Forward Tree method, see Fig. 7(b). Similar to the approach above, one could let the tree grow in the backward direction for $b / 2$ blocks, regardless of the time complexity of the compression function attack. In the forward direction we rely on using the partial-pseudo-preimage on the compression function of cost $2^{c}$ again, now having to call it $2^{b}$ times to have a partial-preimage. Using this, the tree grows in the forwards direction in exactly the same manner as in the backwards direction. Because of the birthday effect, both trees have at least one connection with high probability. The total runtime is $b \cdot 2^{b+c+1}$, the memory requirement is $2^{b / 2}$.

- Tree Method due to Mendel and Rijmen, see Fig. 77(c). In [22] a tree-based method was proposed that has the same runtime and memory requirements as the new graph-based method we are about to introduce in the following section. 


\subsection{A Graph Based Approach}

The meet-in-the-middle method discussed above requires the generation of many partial-preimages for the first part of the preimage and many pseudo-preimages for the second part of the preimage. The new method based on random directed graphs we are about to introduce allows to reduce the number of partialpreimages needed at the beginning and pseudo-preimages needed at the end to 1 , at the cost of a number of partial-pseudo-preimages (each $2^{c}$ ) in between. Hence the name $\mathrm{P}^{3}$ graph method, see also Fig. $\mathbf{7}(\mathrm{c})$. We first outline the proposed method, and give time and memory complexities. Afterwards we discuss and compare it with other methods.

Edges of $\mathbf{P}^{3}$ graph: Using a partial pseudo preimage algorithm, generate $2^{b+1}$ tuples $\left(h_{(i)}, m_{(i)}\right)$, at cost $2^{b+c+1}$. All these tuples, which map $h_{(i)}$ to $f\left(h_{(i)}, m_{(i)}\right)$, can be seen as the $2^{b+1}$ edges of a directed graph consisting of $2^{b}$ nodes. As explained in Appendix $\mathrm{A}$. we expect the majority of those nodes to be part of a large densely interconnected component.

First Message Block, Forward Direction: Using the partial preimage generation method, generate a single tuple $\left(h_{0}, m_{0}\right)$ that hits this component. The expected work is in the order of $2^{b+c}$.

Last Message Block, Backward Direction: Also here, generate a single tuple $\left(h_{x}, m_{x}\right)$ such that $f\left(h_{x}, m_{x}\right)=\mathrm{d}$ and that $h_{x}$ falls into the interconnected part of the graph. The expected work is again in the order of: $2^{b+c}$.

Connection: What remains to be found is a connection (a path) between these nodes (the entry node and the exit node) in the graph. Given the number of edges in the graph, such a path is very likely to exist, as we discuss in detail in Appendix $\mathrm{A}$. Total expected work: $2^{b+c+1}+2^{b+c}+2^{b+c}=2^{b+c+2}$

On Exploiting Precomputation. The computations for constructing the first message block and the $\mathrm{P}^{3}$ graph do not need to be repeated when attacking a different digest. The effort for every additional preimage attack is only $2^{b+c}$.

\subsection{Discussion}

There are a number of useful and distinctive properties of the $\mathrm{P}^{3}$ graph method. Firstly, the graph approach does not impose any structure on the connections of partial-pseudo-preimages, which is an intuitive explanation of the efficiency again compared to the L-Tree and the BF-Tree methods. Secondly, the $\mathrm{P}^{3}$ graph is friendlier towards precomputation: Whereas the full $\mathrm{P}^{3}$ graph (potentially in such a way that the IV of the hash function is one of the nodes) can be precomputed, it is not possible to precompute the backwards tree for the L-Tree and the BF-Tree method. Another advantage of the $\mathrm{P}^{3}$ graph method over all other known methods is that paths (and hence preimages) of almost any length have high probability to exist. There is no upper limit, the lower bound is discussed in 
Appendix A. This property will be useful when dealing with the padding in a preimage attack on the hash function (see Sect 5.1).

One drawback of the $\mathrm{P}^{3}$ graph method can be the higher memory requirements. Storage requirements for all the edges is exponential in the number of bits $b$ that can not be controlled. Hence the runtime gain of the $\mathrm{P}^{3}$ graph method is useful in practice if the compression function attack allows to choose a reasonable small $b$. The $\mathrm{P}^{3}$ graph method allows time/memory tradeoffs that resemble e.g., the BF-Tree method. Space constraints do not allow us to discuss them here. In Table 1 we summarize and compare the meet in the middle approach with the $\mathrm{P}^{3}$ graph method.

\section{$5 \quad$ Putting Everything Together}

We have now set the state to talk about the security margin of the SHA-0 and SHA-1 hash function against the new cryptanalytic methods. We do this by combining the compression function attack from Sect. 3 and the $\mathrm{P}^{3}$ graph method from Sect. 4 .

\subsection{Padding}

So far, we neglected the fact that in a preimage attack on SHA-0 and SHA-1, the padding fixes a part of the input message of the last message block. Hence, without being able to cope with such a restriction, our attack would only be a second preimage attack, but not a preimage attack. We discuss here several possibilities to produce a correctly padded last message block without a first preimage.

- Restrict the Degrees of Freedom in the Compression Function Attack: In order to fix a particular value for the message length, at least the last 65 bits of the last message block need to be fixed. Among them are 25 bits whose freedom is needed in the compression function attack (for both SHA-0 and SHA-1), hence fixing them results (without further optimizations) in a slowdown of the compression function attack by up to a factor of $2^{25}$. In detail, these bits are $M_{14}^{0}, M_{15}^{0 \ldots 4,24 \ldots 31}$ and $M_{16}^{0 \ldots 4,24 \ldots 31}$.

- Expandable Messages: By making sure that every message length can be constructed after the compression function attacks have been performed, almost no additional degrees of freedom need to be spent for a correct padding.

Table 1. Comparison of the meet-in-the-middle approach, various tree approaches, and the $\mathrm{P}^{3}$ graph method. All numbers are exponents of base 2 .

\begin{tabular}{|l|c|c|c|c|c|}
\hline & MITM2 & L(ayered)-Tree & BF-Tree & MR-Tree & P $^{3}$ graph \\
\hline total work & $3 b / 2+c+1$ & $b+c+1+\log _{2}(n-b-c)$ & $b+\log _{2}(b)+c+1$ & $b+c+2$ & $b+c+2$ \\
total mem. & $b$ or less & $n-b-c-1$ & $b / 2$ & $b+1$ & $b+1$ \\
\hline onl. work & $b+c$ & - & - & $b+c$ \\
offl. work & $2 b+c$ & - & - & $b+c+\log _{2}(3)$ \\
memory & $b$ & - & no & $b+c+\log _{2}(3)$ \\
\hline flexible len. & no & no & $b+1$ & no & yes \\
\hline
\end{tabular}


Using any of the following methods will hence return preimages of uncontrollable length. The only two property that the compression function attack needs to have, are as follows. Firstly, to make sure that the end of the message (before the length encoding, i.e., the LSB of $M_{13}$ ) is a ' 1 '. Secondly, make sure that the length is a exact multiple of the block length, i.e., fix the last nine bits of $M_{15}$ to '110111111' (447). In total ten bits need to be fixed for this, which will result (without further optimizations) in a slowdown of the compression function attack by a factor $2^{6}$. In detail, the six crucial bits are $M_{14}^{0}$ and $M_{16}^{0 . .4}$. Possibilities to construct expandable messages are as follows.

- Multicollisions: As soon as the compression function attack has a complexity slightly above the birthday bound $\left(2^{n / 2+\log _{2}(n)}\right)$, the multicollision idea [12] can be used to construct expandable messages [15] without being the bottleneck.

- Flexibility of the $\mathbf{P}^{3}$ graph Method (cycles): In the random directed graph used in the $\mathrm{P}^{3}$ graph method of Sect. 4.2, we expect to have many cycles, also on the path between entry- and exit node. As detailed in Appendix A, we hence expect to find paths of any length longer than some lower bound that connect any entry- and exit node with high probability.

\subsection{Summary of Attacks}

From Sect. 3 we learn that $b_{1}=b_{2}=25$ is a straight-forward choice for the case of SHA-0. Since the method allows us to pick the same bit positions, we also have $b=25$. Since $b_{2} \leq 20$ for SHA-1, we will have to restrict ourselves to $b=20$ in this case. Note that for seriously reduced SHA-0 and SHA-1, less degrees of freedom are of use in the compression function attack, and hence $b$ can be smaller. A quick check in Table 1 will convince the reader that memory requirements will not be a problem in the practical implementation of such an attack, even with the most time efficient $\mathrm{P}^{3}$ graph method.

In order to illustrate our results we consider SHA-0 and SHA-1 reduced to concrete numbers of steps, and give attack complexities in Figure 8. We combine the attacks on the compression function as given in Sect. 3 with the different generic ways of turning them into a preimage attack as outlined in Sect. 4.2. In our implementation of this attack the memory requirements are negligible. Additionally, we also give attack complexities in Table 2. For both SHA-0 and SHA-1, the number of steps for which we list results are chosen as follows. To compare (lack of) resistance against the new attack of similarly reduced primitives, we pick 32 steps in all cases. Additionally, we give results for the highest number of steps for which the attack would be better than the birthday bound and an actual brute force attack, respectively. Our approach takes less than $2^{160}$ hash evaluations for SHA-0 reduced to up to 50 steps and for SHA-1 reduced to up to 45 steps. Note that inverting the hash function also implies the ability to construct a fixed point. 

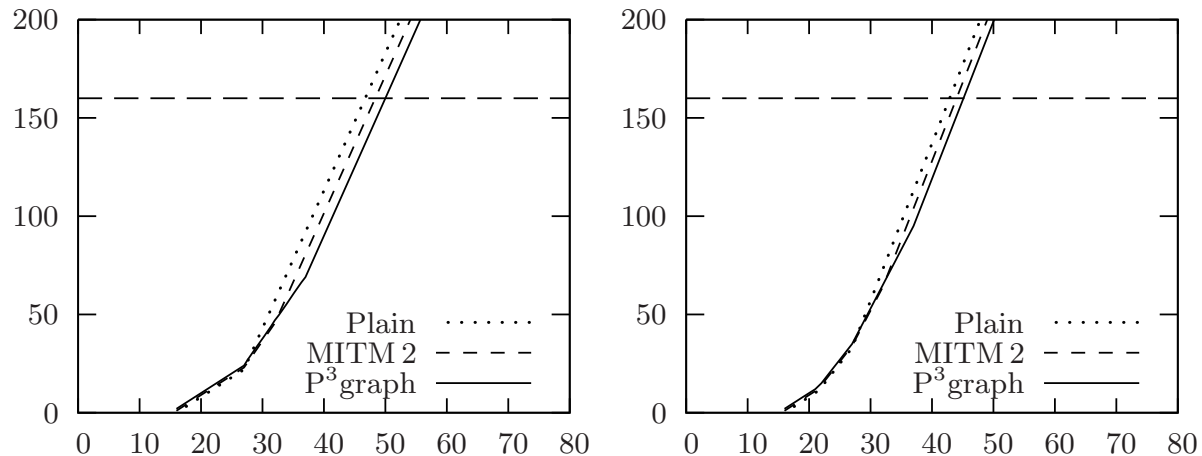

Fig. 8. Complexities of second preimage attacks against reduced SHA-0 (left) and SHA-1 (right). The line 'Plain' refers to a direct preimage attack using only a single block. The line 'MITM 2' refers to a meet-in-the-middle approach where partialpreimages in the forward direction are combined with pseudo-preimages in the backwards direction. The line ' $\mathrm{P}^{3}$ graph' refers to the new graph based method.

Table 2. Exemplification of new preimage attacks on reduced SHA-0 (left table) and SHA-1 (right table). Efforts are expressed in terms of time complexity; memory and communication costs can be considered negligible. For ideal building blocks, all these attacks would require a $2^{160}$ effort. For simplification, the small constant factor between the numbers given here and a naive brute force search is neglected. We give the total runtime for attacking the first target digest; attacks on subsequent targets will be faster.

\begin{tabular}{|c||c|c|c|c||c|c|c|c|}
\hline type of attack & $\begin{array}{c}\text { building } \\
\text { block }\end{array}$ & steps & $b$ & $\begin{array}{c}\text { effort with } \\
\text { new attack }\end{array}$ & $\begin{array}{c}\text { building } \\
\text { block }\end{array}$ & steps & $b$ & $\begin{array}{c}\text { effort with } \\
\text { new attack }\end{array}$ \\
\hline inv. compression f. & SHA-0 & 32 & 25 & $2^{32}$ & SHA-1 & 32 & 20 & $2^{53}$ \\
inv. compression f. & SHA-0 & 38 & 25 & $2^{74}$ & SHA-1 & 35 & 20 & $2^{77}$ \\
inv. compression f. & SHA-0 & 50 & 25 & $2^{158}$ & SHA-1 & 45 & 20 & $2^{157}$ \\
\hline \hline 2nd preimage of hash & SHA-0 & 32 & 12 & $2^{47}$ & SHA-1 & 32 & 10 & $2^{65}$ \\
2nd preimage of hash & SHA-0 & 38 & 25 & $2^{76}$ & SHA-1 & 34 & 14 & $2^{77}$ \\
2nd preimage of hash & SHA-0 & 49 & 25 & $2^{153}$ & SHA-1 & 45 & 20 & $2^{159}$ \\
\hline \hline preimage of hash & SHA-0 & 37 & 25 & $2^{75}$ & SHA-1 & 34 & 17 & $2^{80}$ \\
preimage of hash & SHA-0 & 49 & 25 & $2^{159}$ & SHA-1 & 44 & 20 & $2^{157}$ \\
\hline
\end{tabular}

\section{Conclusions and Outlook}

The first method to construct preimages for SHA-0 and SHA-1 reduced to a nontrivial number of steps (up to 50 out of 80 ) is presented. The impossible message approach we proposed exploits weak diffusion properties in the step transformation and in the message expansion, which allows to divide the work and consider only one or a small number of column at a given time. Both, the impossible message approach, and the $\mathrm{P}^{3}$ graph we introduced to efficiently transform attacks on the compression function to attacks on the hash function, 
are rather generic and await to be applied to other settings and hash functions as well.

Our results shed some light on the security margin offered by SHA-0 and SHA-1 when only preimage attacks are of a concern. However, several aspects of this work suggest that the security margin might be smaller. Let's compare the result of this work on cryptanalytic preimage attacks to the situation of collision search attacks in 2004 and early 2005:

- Step-Reduced Variants: Work on SHA-1 resulted in theoretical collision attacks for up to 58 steps [1, 31]. Our preimage attacks cover slightly less steps but are on a comparable magnitude.

- Degrees of Freedom: Whereas in the most recent collision search attacks on SHA-1 the availability of degrees of freedom is the limiting factor for further improvements, this was of no concern in earlier work. The fact that not all degrees of freedom are used in our new preimage attacks suggests that further improvements are possible.

- Sensitivity for Different Choices of Rotation Constants: The state update transformation of SHA-0 and SHA-1 uses the fixed set of rotation constants $(5,-2)$. A study of the effect of different choices of rotation constants on earlier collision search strategies [26] concluded that already a slightly different choice would impact the performance significantly, although in a complex way. In our attack, we observe a similar situation: The attack complexity directly depends on the used rotation constants and would be lower or higher, depending on the actual choice. The most recent collision search attacks on SHA-1 do not show such a strong dependency on the choice of rotation constants. Again, this suggests that further improvements on the preimage attack presented in this paper is an interesting open problem.

\section{Acknowledgements}

The authors wish to thank Florian Mendel, Adi Shamir, Yiqun Lisa Yin and the anonymous reviewers for their useful comments. The work in this paper has been supported in part by the Fund for Scientific Research (FWO), the Chaire France Telecom pour la sécurité des réseaux, the Secure Information Technology Center-Austria (A-SIT), by the Austrian Science Fund (FWF), project P19863, and by the IAP Programme P6/26 BCRYPT of the Belgian State (Belgian Science Policy).

\section{References}

1. Biham, E., Chen, R., Joux, A., Carribault, P., Lemuet, C., Jalby, W.: Collisions of SHA-0 and Reduced SHA-1. In: Cramer, R. (ed.) EUROCRYPT 2005. LNCS, vol. 3494, pp. 36-57. Springer, Heidelberg (2005)

2. Biham, E., Shamir, A.: Differential Cryptanalysis of DES-like Cryptosystems. In: Menezes, A., Vanstone, S.A. (eds.) CRYPTO 1990. LNCS, vol. 537, pp. 2-21. Springer, Heidelberg (1991) 
3. Black, J., Rogaway, P., Shrimpton, T.: Black-Box Analysis of the Block-CipherBased Hash-Function Constructions from PGV. In: Yung, M. (ed.) CRYPTO 2002. LNCS, vol. 2442, pp. 320-335. Springer, Heidelberg (2002)

4. Bollobás, B.: Random Graphs. Academic Press, London (1985)

5. Bollobás, B.: Modern Graph Theory. Springer, Heidelberg (1998)

6. Chabaud, F., Joux, A.: Differential Collisions in SHA-0. In: Krawczyk, H. (ed.) CRYPTO 1998. LNCS, vol. 1462, pp. 56-71. Springer, Heidelberg (1998)

7. De Cannière, C., Mendel, F., Rechberger, C.: Collisions for 70-Step SHA-1: On the Full Cost of Collision Search. In: Adams, C.M., Miri, A., Wiener, M.J. (eds.) SAC 2007. LNCS, vol. 4876, pp. 56-73. Springer, Heidelberg (2007)

8. De Cannière, C., Rechberger, C.: Finding SHA-1 Characteristics: General Results and Applications. In: Lai, X., Chen, K. (eds.) ASIACRYPT 2006. LNCS, vol. 4284, pp. 1-20. Springer, Heidelberg (2006)

9. Diffie, W., Hellman, M.: Exhaustive cryptanalysis of the NBS Data Encryption Standard. Computer 10(6), 74-84 (1977)

10. Dobbertin, H.: Cryptanalysis of MD4. J. Cryptology 11(4), 253-271 (1998)

11. Erdös, P., Rènyi, A.: On random graphs. Publicationes Mathematicae 6, 290-297 (1959)

12. Joux, A.: Multicollisions in Iterated Hash Functions. Application to Cascaded Constructions. In: Franklin, M.K. (ed.) CRYPTO 2004. LNCS, vol. 3152, pp. 306-316. Springer, Heidelberg (2004)

13. Joux, A., Peyrin, T.: Hash Functions and the (Amplified) Boomerang Attack. In: Menezes, A. (ed.) CRYPTO 2007. LNCS, vol. 4622, pp. 244-263. Springer, Heidelberg (2007)

14. Kelsey, J., Kohno, T.: Herding Hash Functions and the Nostradamus Attack. In: Vaudenay, S. (ed.) EUROCRYPT 2006. LNCS, vol. 4004, pp. 183-200. Springer, Heidelberg (2006)

15. Kelsey, J., Schneier, B.: Second Preimages on n-Bit Hash Functions for Much Less than $2^{\mathrm{n}}$ Work. In: Cramer, R. (ed.) EUROCRYPT 2005. LNCS, vol. 3494, pp. 474-490. Springer, Heidelberg (2005)

16. Lamberger, M., Pramstaller, N., Rechberger, C., Rijmen, V.: Second Preimages for SMASH. In: Abe, M. (ed.) CT-RSA 2007. LNCS, vol. 4377, pp. 101-111. Springer, Heidelberg (2006)

17. Leurent, G.: MD4 is Not One-Way. In: Nyberg, K. (ed.) FSE 2008. LNCS. Springer, Heidelberg (to appear, 2008)

18. Mendel, F., Pramstaller, N., Rechberger, C.: A (Second) Preimage Attack on the GOST Hash Function. In: Nyberg, K. (ed.) FSE. LNCS, vol. 5086, pp. 224-234. Springer, Heidelberg (2008)

19. Mendel, F., Pramstaller, N., Rechberger, C., Kontac, M., Szmidt, J.: Cryptanalysis of the GOST Hash Function. In: Wagner, D. (ed.) Proceedings of CRYPTO 2008. LNCS. Springer, Heidelberg (to appear, 2008)

20. Mendel, F., Pramstaller, N., Rechberger, C., Rijmen, V.: The Impact of Carries on the Complexity of Collision Attacks on SHA-1. In: Robshaw, M.J.B. (ed.) FSE 2006. LNCS, vol. 4047, pp. 278-292. Springer, Heidelberg (2006)

21. Mendel, F., Rechberger, C., Rijmen, V.: Update on SHA-1. In: Rump Session of CRYPTO 2007 (2007)

22. Mendel, F., Rijmen, V.: Weaknesses in the HAS-V Compression Function. In: Nam, K.-H., Rhee, G. (eds.) ICISC 2007. LNCS, vol. 4817, pp. 335-345. Springer, Heidelberg (2007) 
23. Morita, H., Ohta, K., Miyaguchi, S.: A Switching Closure Test to Analyze Cryptosystems. In: Feigenbaum, J. (ed.) CRYPTO 1991. LNCS, vol. 576, pp. 183-193. Springer, Heidelberg (1992)

24. National Institute of Standards and Technology. NIST's Policy on Hash Functions (2006), http://csrc.nist.gov/groups/ST/hash/policy.html

25. National Institute of Standards and Technology (NIST). FIPS-180-2: Secure Hash Standard (August 2002), http://www.itl.nist.gov/fipspubs/

26. Pramstaller, N., Rechberger, C., Rijmen, V.: Impact of Rotations in SHA-1 and Related Hash Functions. In: Preneel, B., Tavares, S.E. (eds.) SAC 2005. LNCS, vol. 3897, pp. 261-275. Springer, Heidelberg (2006)

27. Preneel, B., Govaerts, R., Vandewalle, J.: Hash Functions Based on Block Ciphers: A Synthetic Approach. In: Stinson, D.R. (ed.) CRYPTO 1993. LNCS, vol. 773, pp. 368-378. Springer, Heidelberg (1994)

28. Quisquater, J.-J., Delescaille, J.-P.: How Easy is Collision Search. New Results and Applications to DES. In: Brassard, G. (ed.) CRYPTO 1989. LNCS, vol. 435, pp. 408-413. Springer, Heidelberg (1990)

29. Rechberger, C., Rijmen, V.: On Authentication with HMAC and Non-random Properties. In: Dietrich, S., Dhamija, R. (eds.) FC 2007 and USEC 2007. LNCS, vol. 4886, pp. 119-133. Springer, Heidelberg (2007)

30. Rechberger, C., Rijmen, V.: New Results on NMAC/HMAC when Instantiated with Popular Hash Functions. Journal of Universal Computer Science (JUCS), Special Issue on Cryptography in Computer System Security 14(3), 347-376 (2008)

31. Rijmen, V., Oswald, E.: Update on SHA-1. In: Menezes, A. (ed.) CT-RSA 2005. LNCS, vol. 3376, pp. 58-71. Springer, Heidelberg (2005)

32. Stevens, M., Lenstra, A.K., de Weger, B.: Chosen-Prefix Collisions for MD5 and Colliding X.509 Certificates for Different Identities. In: Naor, M. (ed.) EUROCRYPT 2007. LNCS, vol. 4515, pp. 1-22. Springer, Heidelberg (2007)

33. Wang, X., Yin, Y.L., Yu, H.: Finding Collisions in the Full SHA-1. In: Shoup, V. (ed.) CRYPTO 2005. LNCS, vol. 3621, pp. 17-36. Springer, Heidelberg (2005)

34. Wang, X., Yu, H.: How to Break MD5 and Other Hash Functions. In: Cramer, R. (ed.) EUROCRYPT 2005. LNCS, vol. 3494, pp. 19-35. Springer, Heidelberg (2005)

35. Yu, H., Wang, G., Zhang, G., Wang, X.: The Second-Preimage Attack on MD4. In: Desmedt, Y.G., Wang, H., Mu, Y., Li, Y. (eds.) CANS 2005. LNCS, vol. 3810, pp. 1-12. Springer, Heidelberg (2005)

\section{A Some Useful Properties of Random Graphs}

In this appendix, we briefly review some properties of random graphs which are relevant to the graph based approach proposed in Sect.4.2. For a more rigorous and comprehensive treatment of random graph theory we refer to [4, 11] and [5, Chapt. VII.5].

\section{A.1 Following Edges in a Random Directed Graph}

Let $G$ be a large directed graph consisting of $n$ nodes and $m=c \cdot n$ randomly selected edges. On average, each node has $c$ outgoing edges, and we denote the probability that a given ordered pair of nodes is connected by an edge by:

$$
p_{c}=\frac{m}{n^{2}}=\frac{c}{n} .
$$


Let us now study what happens when we start from an arbitrary node $a$ and construct sets of nodes $S_{0}, S_{1}, S_{2}, \ldots$ where $S_{0}=\{a\}$, and $S_{i}$ contains all nodes that can be reached from $a$ in exactly $i$ hops. If we eventually end up with an empty set, the initial node $a$ is called a "dying" node. In the opposite case, $a$ is said to "explode". Clearly, if there exists an edge from $a$ to $b$, and $b$ is an exploding node, then $a$ must be exploding as well. Conversely, a node $a$ can only die if none of the $n$ nodes in the graph are both connected to $a$ and exploding. Hence, the probability $p_{e}$ that a node explodes must satisfy:

$$
1-p_{e}=\left(1-p_{c} \cdot p_{e}\right)^{n} \approx e^{-c \cdot p_{e}} .
$$

From this expression we can deduce that $p_{e}$ must necessarily be 0 as long as $c \leq 1$. However, when $c>1$, the equation $1-x=e^{-c \cdot x}$ does have a non-zero (and positive) solution, which we will refer to as $\gamma(c) 1$

Assuming that the sets $S_{i}$ reach some moderately large size (i.e., $a$ does not die), we can write a simple recursive relation between the expected sizes $E\left(\left|S_{i}\right|\right)$ of successive sets by computing the probability that an arbitrary node is connected to at least one node of $S_{i}$ :

$$
E\left(\left|S_{i+1}\right|\right)=n \cdot\left[1-\left(1-p_{c}\right)^{E\left(\left|S_{i}\right|\right)}\right] \approx n \cdot\left[1-e^{-c \cdot E\left(\left|S_{i}\right|\right) / n}\right] .
$$

Note that we can apply the same reasoning to obtain an almost identical recursive relation between successive values of $E\left(\left|S_{0} \cup S_{1} \cdots S_{i}\right|\right)$. By filling in $i=\infty$, we find that the expected size of the sets converges to:

$$
E\left(\left|S_{\infty}\right|\right) \approx E\left(\left|S_{0} \cup S_{1} \cdots S_{\infty}\right|\right) \approx n \cdot \gamma(c)
$$

\section{A.2 Connecting Two Given Nodes}

In the previous section, we argued that a node $a$ explodes with probability $p_{e}=\gamma(c)$, and that a fraction $\gamma(c)$ of all nodes can be reached from it if it does. Similarly, if $a$ dies, it can be shown that only a negligible fraction of nodes will be reached. The probability $p_{p}$ that two given nodes $a$ and $b$ are connected by a path is hence:

$$
p_{p}=\gamma(c)^{2}
$$

In the context of the attack proposed in this paper, we are interested in the expected number of random edges $\bar{m}$ that need to be added to a graph in order to find a path between two given nodes $a$ and $b$. Suppose our current graph has $m>n$ edges. In that case we know that with probability $1-\gamma(m / n)^{2}$ there will be no path between $a$ and $b$, in which case we will need at least one more edge. Repeating this reasoning, we find;

$$
\bar{m} \approx n+\sum_{m=n}^{n^{2}}\left[1-\gamma(m / n)^{2}\right] .
$$

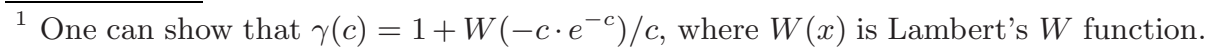


We can approximate this sum by an integral, and after a few changes of variables, we eventually obtain:

$$
\begin{aligned}
\bar{m} & \approx n+n \cdot \int_{1}^{\infty}\left[1-\gamma(c)^{2}\right] d c \\
& =n+n \cdot \int_{0}^{1}\left(1-\gamma^{2}\right) \cdot \frac{d c}{d \gamma} d \gamma \\
& =2 \cdot n .
\end{aligned}
$$

This result, which states that, in order to connect two given nodes, we need on average twice as many edges as nodes (i.e., $c=2$ ), is the main property used in Sect. 4.2 .

\section{A.3 Path Lengths}

If we want to apply our graph based attack to a hash function which includes the message length in the padding block, then we not only need to make sure that there exists a path between two given nodes; we would also like to know in advance how long this path will be.

In order to estimate how many nodes can be reached for a fixed path length, we need to solve the recursive relation of (2). A closed form solution probably does not exist, but we can find a very good approximation:

$$
E\left(\left|S_{i}\right|\right) \approx n \cdot \gamma \cdot \frac{\left[\alpha^{2 \cdot(i-\delta)}+1\right]^{\beta}}{\alpha^{(i-\delta)}+1},
$$

where $\alpha=c \cdot(1-\gamma), \alpha^{2 \cdot \beta-1}=c$, and $n \cdot \gamma \cdot c^{-\delta}=1$. For $c=2$, we find that $\gamma=0.80, \alpha=0.41, \beta=0.12$, and

$$
\delta=\frac{1}{\log _{2} c} \cdot\left(\log _{2} n+\log _{2} \gamma\right)=\log _{2} n-0.33 .
$$

We can now compute the minimal path length $l$ for which we expect that $S_{l}$ includes all reachable nodes (i.e., $\left.S_{l}=S_{\infty}\right)$. By solving the inequality $E\left(\left|S_{\infty}\right|\right)-$ $E\left(\left|S_{l}\right|\right)<1$, we obtain:

$$
l>\left[\frac{1}{\log _{2} \alpha}-\frac{1}{\log _{2} c}\right] \cdot\left(\log _{2} n+\log _{2} \gamma\right)=1.77 \cdot \log _{2} n-0.58 .
$$

In other words, if we are given a random graph with $n$ nodes and $2 \cdot n$ edges, and if this graph connects two arbitrary nodes $a$ and $b$ (this occurs with probability $\left.\gamma^{2}=0.63\right)$, then we expect to find paths from $a$ to $b$ of length $l$ for any $l$ exceeding $1.77 \cdot \log _{2} n$.

\section{B Proof-of-Concept Examples}

As a proof-of-concept, we give examples of an implementation of the described methods. We chose two examples. The first is a preimage for the 33-step SHA-0 compression function. The second is also a second preimage of a (roughly) $1 \mathrm{~KB}$ ASCII text for the 31-step SHA-0 hash function, using the $\mathrm{P}^{3}$ graph method. 


\section{B.1 A Preimage for the 33-Step Compression Function of SHA-0}

As a proof-of-concept, we consider the compression function of SHA-0 reduced to 33 steps. In Figure 9 we give a preimage for the all- 1 output. $A_{-4} \ldots A_{0}$ and $W_{0} \ldots W_{15}$ represent the input to the compression function. Computing $A_{-4}+A_{29} \ldots A_{0}+A_{33}$ results in the all-1 output.

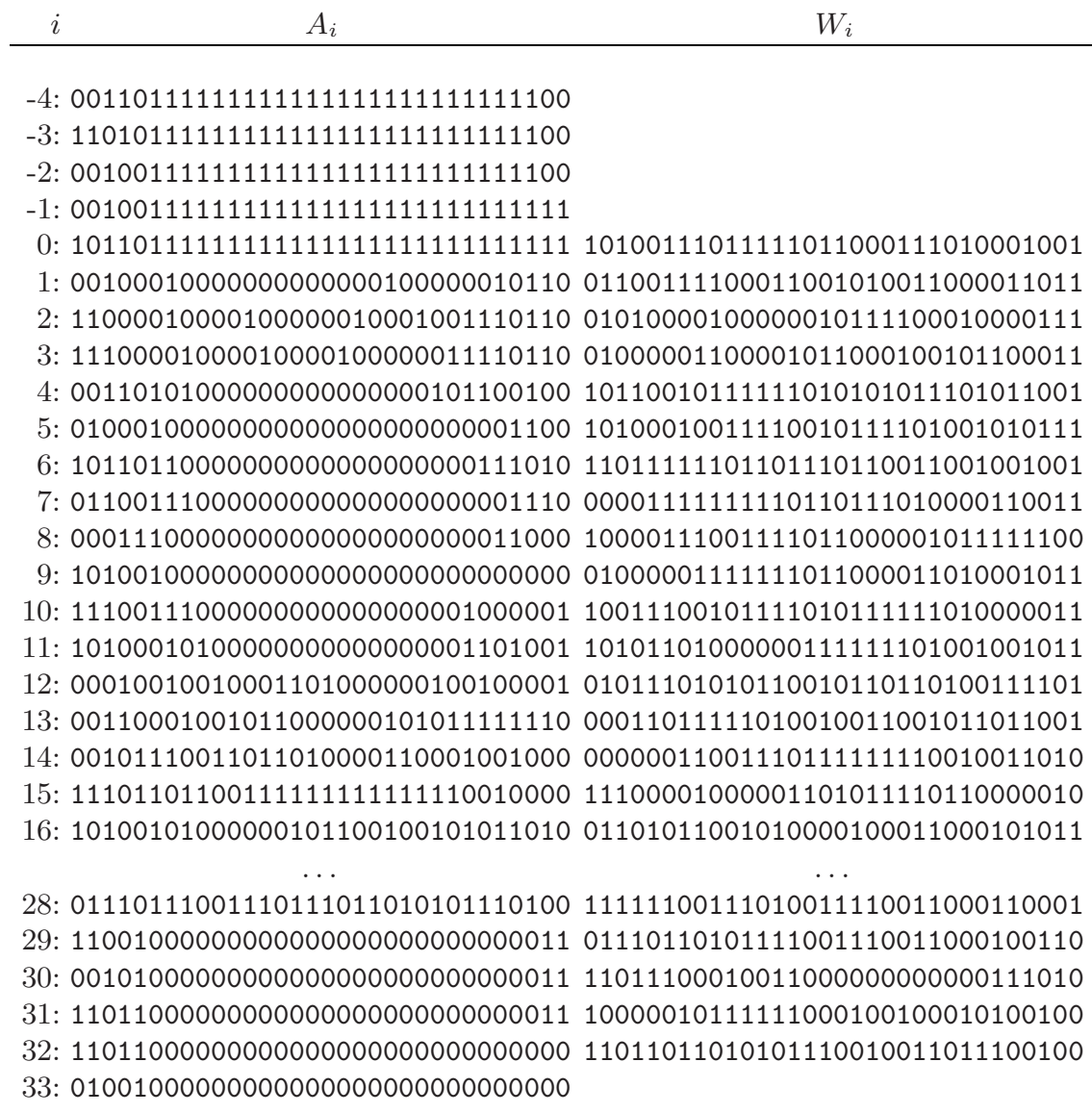

Fig. 9. A preimage of the all-1 output for the 33-step SHA-0 compression function 
0000000: 416c $6963652077617320626567696 \mathrm{e} 6 \mathrm{e}$ 0000010: 696 e $6720746 f 20676574207665727920$ 0000020: $7469726564206 f 66 \quad 2073697474696 e 67$ 0000030: $2062792068657220736973746572 \quad 206 \mathrm{f}$ 0000040: 6e20 7468 6520 6261 6e6b 2c20 616e 6420 0000050: 6f66 20686176 696e 6720 6e6f 7468 696e 0000060: $6720746 f 2064$ 6f3a 206f 6e63 6520 6f72 0000070: 20747769636520736865206861642070 0000080: 656570656420 696e 746f 207468652062 0000090: $6 \mathrm{f} 6 \mathrm{f} \quad 6 \mathrm{~b} 2068657220736973746572 \quad 2077$ 00000a0: $61732072656164696 \mathrm{e} 67 \quad 2 \mathrm{c} 2062757420$ 00000b0: 697420686164 206e 6f20 706963747572 00000c0: 6573 206f $7220636 f 6 e 76657273617469$ 00000d0: 6f6e 7320 696e 2069742 c 2060616 e 6420 00000e0: 77686174206973207468652075736520 00000f0: 6f66 $206120626 f 6 f \quad 6 b 2 c \quad 27207468 \quad 6 f 75$ 0000100: $67687420416 \mathrm{c} 6963652060776974686 \mathrm{f}$ 0000110: $7574207069637475726573206 f 722063$ 0000120: $6 \mathrm{f} 6 \mathrm{e} 766572736174$ 696f 6e $3 \mathrm{f} 2720536 \mathrm{f}$ 0000130: 20736865207761732063 6f6e 73696465 0000140: $72696 \mathrm{e} 67 \quad 20696 \mathrm{e} 20686572206 f 77 \quad 6 e 20$ 0000150: 6d69 6e64 202861732077 656c 6c20 6173 0000160: $2073686520636 f 756 c 64$ 2c20 666f 7220 0000170: 74686520 686f $7420646179206 \mathrm{~d} 616465$ 0000180: $20686572206665656 \mathrm{c} 20766572792073$ 0000190: $6 \mathrm{c} 6565707920616$ e 6420737475706964 00001a0: 292c 2077686574686572207468652070 00001b0: $6 \mathrm{c} 656173757265206 \mathrm{f} 66$ 206d 616b 696e 00001c0: $6720612064616973792 d 636861696 \mathrm{e} 20$ 00001d0: $776 f$ 756c 642062652077 6f72 74682074 00001e0: $68652074726 f 75626 \mathrm{c} 65$ 206f 66206765 00001f0: 7474 696e $6720757020616 e 6420706963$ 0000200: $6 \mathrm{~b} 696 \mathrm{e} 67207468652064616973696573$ 0000210: 2c20 7768 656e 207375646465 6e6c 7920 0000220: 61205768697465205261626269742077 0000230: 697468207069 6e6b 2065796573207261 0000240: 6e20 636c 6f73 6520627920686572 2e20 0000250: 54686572652077617320 6e6f $7468696 \mathrm{e}$ 0000260: $6720736 \mathrm{f} 2056455259207265$ 6d61 726b 0000270: 6162 6c65 $20696 \mathrm{e} 2074686174$ 3b20 6e6f 0000280: $722064696420416 \mathrm{c} 696365207468696 \mathrm{e}$ 0000290: $6 \mathrm{~b} 2069742073$ 6f20 56455259 206d 7563 00002a0: 6820 6f75 7420 6f66 2074686520776179 00002b0: 2074 6f20 686561722074686520526162 00002c0: $6269742073617920746 f 20697473656 c$ 00002d0: 662c $20604 f 68206465617221204 f 6820$ 00002e0: 64656172212049207368 616c $6 c 206265$ 00002f0: 206c $617465212720287768656 \mathrm{e} 207368$
Alice was beginn ing to get very tired of sitting by her sister 0 $\mathrm{n}$ the bank, and of having nothin $g$ to do: once or twice she had $p$ eeped into the $\mathrm{b}$ ook her sister w as reading, but it had no pictur es or conversati ons in it, 'and what is the use of a book,' thou ght Alice 'witho ut pictures or $c$ onversation?' So she was conside ring in her own mind (as well as she could, for the hot day made her feel very $s$ leepy and stupid ), whether the $p$ leasure of makin $\mathrm{g}$ a daisy-chain would be worth $t$ he trouble of ge tting up and pic king the daisies , when suddenly a White Rabbit w ith pink eyes ra n close by her. There was nothin g so VERY remark able in that; no $r$ did Alice thin $k$ it so VERY muc $h$ out of the way to hear the Rab bit say to itsel $f$, 'Oh dear! Oh dear! I shall be late!' (when sh

Fig. 10. 31-round SHA-0: original message (part 1) 
0000300: $65207468 \quad 6 f 756768 \quad 74206974 \quad 206 f 7665$ 0000310: $72206166 \quad 74657277 \quad 6172 \quad 6473 \quad 2 c 206974$ 0000320: 206f $6363757272656420746 f 20686572$ 0000330: $207468617420736865206 f 7567687420$ 0000340: 746f $20686176 \quad 6520776 f \quad 6 e 6465726564$ 0000350: $2061742074686973 \quad 2 c 20627574206174$ 0000360: 207468652074 696d $6520697420616 c 6 c$ 0000370: $207365656 \mathrm{~d} 6564207175697465206 \mathrm{e} 61$ 0000380: $74757261 \quad 6 c 29 \quad 3 b 20627574207768 \quad 656 e$ 0000390: 20746865205261626269742061637475 00003a0: 616c 6c79 2054 4f4f 4b20 412057415443 00003b0: 4820 4f55 $54204 f 4620495453 \quad 20574149$ 00003c0: 5354 434f 4154 2d50 4f43 4b45 542c 2061 00003d0: 6e64 206c 6f6f 6b65 $642061742069742 c$ 00003e0: $20616 e 64207468656 e 20687572726965$ 00003f0: 6420 6f6e 2c20 416c 6963652073746172 0000400: $74656420746 f 2068657220666565742 c$ 0000410: 2066 6f72 $20697420 \quad 666 \mathrm{c} \quad 617368656420$ 0000420: $6163726 f 737320686572$ 206d 696e 6420 0000430: 746861742073686520686164 206e 6576 0000440: 657220626566 6f72 65207365 656e 2061 0000450: $207261626269742077697468 \quad 20656974$ 0000460: $6865722061207761697374636 f 61742 d$ 0000470: $706 f$ 636b 6574 2c20 6f72 206120776174 0000480: 63682074 6f20 7461 6b65 206f $7574206 f$ 0000490: 66206974 2c20 616e 64206275726 e 696e 00004a0: 67207769746820637572 696f 73697479 00004b0: 2c20 7368 $652072616 \mathrm{e} 206163726 \mathrm{f} 7373$ 00004c0: $20746865206669656 c 64206166746572$ 00004d0: 2069 742c $20616 e 642066$ 6f72 7475 6e61 00004e0: 7465 6c79 20776173 206a $7573 \quad 7420$ 696e 00004f0: 2074 696d $6520746 f 2073656520697420$ 0000500: 706f 7020 646f 776e 2061 206c 61726765 0000510: $207261626269742 d$ 686f 6c65 $20756 e 64$ 0000520: $6572207468652068656467652 \mathrm{e} 20496 \mathrm{e}$ 0000530: 2061 6e6f 74686572 206d 6f6d 656e 7420 0000540: 646f 776e 2077 656e 7420 416c 69636520 0000550: 6166746572206974 2c20 6e65 76657220 0000560: 6f6e 63652063 6f6e $7369646572696 e 67$ 0000570: 2068 6f77 2069 6e20 $74686520776 f \quad 726 c$ 0000580: $64207368652077617320746 f 20676574$ 0000590: 206f 757420616761696 e 2e0a e thought it ove $r$ afterwards, it occurred to her that she ought to have wondered at this, but at the time it all seemed quite na tural); but when the Rabbit actu ally TOOK A WATC $\mathrm{H}$ OUT OF ITS WAI STCOAT-POCKET, a nd looked at it, and then hurrie d on, Alice star ted to her feet, for it flashed across her mind that she had nev er before seen a rabbit with eit her a waistcoatpocket, or a wat ch to take out o $f$ it, and burnin g with curiosity , she ran across the field after it, and fortuna tely was just in time to see it pop down a large rabbit-hole und er the hedge. In another moment down went Alice after it, never once considering how in the worl d she was to get out again.

Fig. 11. 31-round SHA-0: original message (part 2) 
0000000: 6093 e793 8844 423f cf3e 414034795078 0000010: f8ac 0a92 7e6a 1956 d8b7 b004 1bf9 027f 0000020: 13fd 7b20 5cbd 783c 9b3d 78d2 e0bd 8106 0000030: fee5 2a1d 8efe 23eb 6bd8 $7621354 f$ 0c9c 0000040: 9b86 3bbf 6469 db87 b11d 9195 707d 3f5a 0000050: 277b 582e 44fa 9440 a57c be61 14bc 7c39 0000060: aabc 785e 3c7d 85ef 35bd 855d 1b7d 84fd 0000070: a7d6 c497 a55a d1ae 21ea 5210 19cc f5e1 0000080: b6a5 86d7 e20e 085d e7ab ab81 dd74 ffad 0000090: 6a33 7421 b5cf $5 f a 2$ c709 48b3 836d 6f2a 00000a0: 8d3d 7e50 eefd 793c 2cbd 84ea d83d 78bc 00000b0: 7d7b 64a9 483c $18 f 3$ f559 a0d5 bf69 d5f8 00000c0: 5e7d 920f 9cbe 10a2 0d5d 5bb1 453d 7b31 00000d0: d03d 7f7f fe6d 019b 5 fa4 fed5 fbf5 79dd 00000e0: 37bd 7ced ddfd 79aa 18fd 7da7 063d 8622 00000f0: ece1 65d6 0372 499e 9c7c 84725267 8c88 0000100: fa9e 8747 255d a7e9 cafd 73dd b87d 3785 0000110: b63d 3c42 2e35 $3292771 b$ 690c a41b $77 f 1$ 0000120: abfd 84fa d93d 8646 9c3d 7774 b23d 7c79 0000130: aef9 1db8 c192 413e d8ef 6d8b b39e f536 0000140: Ofa1 c66f 3ffd 955e 6f3b c780 3265 afa6 0000150: 76ac 6b63 fa32 $6784510 \mathrm{~b} 5 \mathrm{c} 5 \mathrm{~d}$ cd0d 5413 0000160: babd 6b15 c5fd 7cab b17d 7c12 a97d 7d5a 0000170: d313 a994 f376 99d2 49b4 e6df 154a 5d84 0000180: 38a0 0a47 d12e 07c9 9065 778b 1b7d 7f34 0000190: 54bc dbfd 2cb4 96c2 0ebb 3db1 8afb 8442 00001a0: 74bd 7b59 25fd 7951 86fd 7ff1 717d 78be 00001b0: 5357 37b3 65247861 6ab2 ec05 8f4c 966e 00001c0: ec5d 8b9f 2d7d 6fb7 f36b fba1 eb6d 7b34 00001d0: bdc5 8179 08c5 5b61 89fd 3b15 2b7d 59ab 00001e0: f07d 7fcc 36fd 7c85 3cbd 7eac 45fd 85c4 00001f0: 752d aeef df79 9808 a886 8285 a5dd ff34 0000200: 5c8d 9e8f b2ba 8079 167d 657a c33d 43bc 0000210: 1db9 76d0 e3e9 70df 986d 7c1e 657d 8363 0000220: 613d 7750 3e3d 7944 fa7d 77 a5 373d 7765 0000230: c560 ac62 e5b2 47dd 01fe aebe e8ac e99a 0000240: 887d 930f 5f7c ofc3 f789 7790 de7d 7f71 0000250: b4bd 7ba9 4d3d 6c8a 1579 75b8 c439 84d2 0000260: 513d 7b27 a3bd 7f43 357d 7fa9 e9bd 7704 0000270: ff1d 6a35 02bd 38592703 d027 49155452 0000280: dd05 9eb7 577a $826301 \mathrm{a} 2$ a46f d8bd 5daa 0000290: eebd 72a2 21db 732a 98b3 f657 d033 fb18 00002a0: 987d $82 f 5 \mathrm{f} 2 \mathrm{bd} 7 \mathrm{c08} 2 \mathrm{dfd} 85 \mathrm{c} 8$ 38fd $82 \mathrm{ca}$ 00002b0: 5939 ee8e 140f 5b3d 0cc9 9c81 9c92 5965 00002c0: 3b9d 96af 8b47 7d9f e2ff 8392 c6ac ff71 00002d0: b5f3 81bd d482 750b 5749 f1aa 4cfc e77a 00002e0: b1fd 7ead e23d 7900 aabd $7 f 55$ 3cbd $83 f 5$ 00002f0: 97bb e4dd 6941 50cd 567f 37d0 3e5c 9e26

$$
\begin{aligned}
& \text { '.... DB? .>A@4yPx } \\
& \ldots \sim \text { j.V...... } \\
& \ldots\{\backslash . \mathrm{x}<.=\mathrm{x} . \ldots . \\
& \text {..*...\#.k.v!50. . } \\
& \text {..;.di.....p\}?Z } \\
& \text {, \{X.D..@.|.a..|9 } \\
& \left.\left.\left.\ldots x^{\wedge}<\right\} \ldots 5 . .\right] .\right\} \ldots \\
& \text {....Z.. !.R.... } \\
& \text {.....t...t.t. } \\
& \text { j3t!.....H. .mo* } \\
& .=\sim \mathrm{P} \ldots \mathrm{y}<, \ldots \mathrm{x}=\mathrm{x} \text {. } \\
& \}\{\text { d. H<... Y...i. } \\
& -\} \ldots . . .][. E=\{1 \\
& .=\ldots \mathrm{m} . . . \mathrm{yy} \text {. } \\
& 7 . \mid \ldots y \ldots\} . .=. " \\
& \text {..e..rI..।.rRg. } \\
& \text {...G\%] ....s..\}7 . } \\
& \text {. }=<\text { B. 52.w.i...w. } \\
& \ldots . .=. F .=w t .=\mid y \\
& \ldots . . . A>\ldots m \ldots 6 \\
& \text {...o?..^o; . .2e. . } \\
& \text { v.kc.2g.Q. \]..T. } \\
& \text {..k...|.\}|..\}\}Z } \\
& \text {....V...I...J] . } \\
& \text { 8..G.....ew..\}. } 4 \\
& \mathrm{~T} \ldots, \ldots . . . . \mathrm{B} \\
& \text { t. }\{Y \% \cdot y Q \ldots q\} x \text {. } \\
& \text { SW7.e\$xaj.....L.n } \\
& \text {.] ..-\}o..k...m\{4 } \\
& \text {... y. [a. . ; . + }\} \text { Y. } \\
& \text {.\}. 6.|.<. . E... } \\
& \mathrm{u}-\ldots \mathrm{y} \ldots \ldots .4 \\
& \text { \....y. \}ez. }=\text { C. } \\
& \text {..v...p..ml.e\}.c } \\
& a=w P>=y D .\} w .7=w e \\
& \text {.'b..G....... } \\
& \text {.\}... } \mid \ldots . \text { w..\}.q } \\
& \text {.. }\{. M=1 \ldots y u .9 . \text {. } \\
& Q=\left\{{ }^{\prime} \ldots \mathrm{C} 5\right\} \ldots . \mathrm{W} \text {. } \\
& \text {..j5..8Y'..'I.TR } \\
& \text {...WW.c.....]. } \\
& \text {..r.!.s*...W.3. . } \\
& \text {.\}.........8. } \\
& \mathrm{Y} 9 \ldots[=\ldots . . \mathrm{Ye} \\
& \text {;...G } \ldots \mathrm{G} \ldots . \mathrm{q} \\
& \text {....u.WI. .L..z } \\
& \ldots \sim \ldots=y \ldots \text {. . U< . . } \\
& \text {....iAP.V.7.>\.\& }
\end{aligned}
$$

Fig. 12. 31-round SHA-0: second preimage (part 1) 
0000300: 7a23 d3cf cdbc 6851 fc6b $6 f d c 0 a 73$ e75c 0000310: 5c53 e94b c211 c83c 9d3b 59c7 77fd 7a5a 0000320: 9afd 7b0b 883d 835f c8fd 7f30 98bd 7f34 0000330: 570a e920 9bc7 4e38 9d9f 7faa 7e51 9dbd 0000340: Of0c c697 20e5 9f98 9c99 fff8 442d 7383 0000350: 583a 2e86 7bc5 a5a9 48e1 57da 0675 61ce 0000360: 1a3d 78d0 23bd 7ac5 24fd 804e 473d 7aa0 0000370: b7c3 6cdc 9ce1 $225187 d 2$ dbef 4739 a47c 0000380: 9d15 92a7 4a9c bcc5 74a9 579c 41dd 7e99 0000390: a8db 7a99 398f 4864 1fa4 54bd 9d6c 7c8e 00003a0: 57bd 7ac7 12fd 84b9 703d 7a02 9cbd 7c37 00003b0: f88f b361 8ec1 1971 f419 9d71 beb2 f4ca 00003c0: 1c42 eccf 31e1 3783 3e6d bf75 $376583 a 6$ 00003d0: 41cc $5 f 17$ c588 0436 df79 4dd9 fafd $752 f$ 00003e0: 353d 7fcc fffd 79e5 057d 7cc1 c93d 84b5 00003f0: 9080 9f98 $75 f 5$ c427 c6d3 ffbb $2 \mathrm{~d} 55$ 00d0 0000400: 3c01 d6c7 410b 7bcd 8d7c f79e c27d 7b5c 0000410: f6dc 7047 4bd6 6e66 2ab7 84a2 2e7d 8676 0000420: b1fd 795b dbbd 7e58 043d 82bf 9b3d 836b 0000430: fbc6 $048529 f 25213$ 6b02 b802 3b6a 30df 0000440: fa7d 8887 177d 4027 298e 7ba9 145b 7aed 0000450: 303d 8219 9cbd 7c5f 1cf9 36b5 b439 3dee 0000460: b63d 76d4 9bfd 7b6c bdbd 83b8 7e3d 8463 0000470: 93b0 32ab c928 2966 29aa ae16 6ec5 9ad0 0000480: 067e 86bf 306d 7b87 f77d ffb8 446d 7bcf 0000490: 143d 35b6 e879 39cf d7b9 5c05 79bd 571f 00004a0: 2cfd $86404 f 7 d 80 d 7$ bf3d 85b5 7d7d 7e35 00004b0: ef2e $825595 \mathrm{e} 183616086$ 946e e1ce 3da9 00004c0: e88c eab7 23f1 0da3 261b 7baf ce35 6bae 00004d0: 2f39 e040 12a1 a732 463d 693f d915 7566 00004e0: bfbd 7d9d 853d 7bee f6bd 7d1e 1e3d 7afe 00004f0: 8ecb 8c22 62eb 7e25 7d3d fbc1 0f75 350d 0000500: d281 c797 $9775600077 \mathrm{df} 9 \mathrm{f} 953737 \quad 7 \mathrm{fbb}$ 0000510: 485c 79e1 0b9c 75850344 efea 56e 4 f0e6 0000520: 4b7d 78a6 2efd 7fc3 f03d 80c3 3f3d 827a 0000530: 30c8 30471144 d3a9 104a 7c41 39474120 0000540: 49a0 8a9f 5c1d 026b e885 637427758269 0000550: cb7d 017c fcb4 c107 50fb 6c2e 37bb 71a6 0000560: eb7d 821c d3bd 8633 6ffd 7cbd 81fd 77e7 0000570: b2c4 fef3 1c48 7d72 136a 2995 0afe 99d5 0000580: $64207368652077617320746 f 20676574$ 0000590: 206f $757420616761696 \mathrm{e} 2 \mathrm{e} 0 \mathrm{a}$

z\#...hQ.ko..s. $\backslash$ $\backslash \mathrm{S} . \mathrm{K} . .<. ; \mathrm{Y} . \mathrm{W} . \mathrm{ZZ}$ $\ldots\{\ldots=. \ldots 0 \ldots 4$ W. . . N8 ... Q Q . ........ $\ldots$ D-s. $\mathrm{X}: \ldots\{\ldots$..W..ua. $.=\mathrm{x} . \# \cdot \mathrm{z} \cdot \$ . . \mathrm{NG}=\mathrm{z}$. ....."Q...G9.। ....J..t.W.A. . .z.9.Hd.T..Il. W. $z \ldots . p=z \ldots \mid 7$ ...a......... .B. .1.7.>m.u7e. . A._...6.yM...u/ $5=\ldots y ..\} \mid . .=$. ...u...'...-U. <...A. $\{\ldots \mid \ldots\}\{\backslash$ . pGK.nf*...\}. $v$ $\ldots y[\ldots \sim \mathrm{X} .=\ldots=. \mathrm{k}$ ....).R.k...;j0. . $\left.\} \ldots\} \Theta^{\prime}\right) .\{\ldots[z$. $0=\left.\ldots\right|_{-} .6 .9=$. .$=\mathrm{v} \ldots\{1 \ldots \sim=. \mathrm{c}$ .2.()f)...n. . . Om \{..\} . Dm $\{$. .$=5 \ldots y 9 \ldots \backslash \cdot \mathrm{y} \cdot \mathrm{W}$. , .๑0\}... ..\} $\}^{\sim} 5$ ...U...a'..n. . . W..\#...\&.\{..5k. 19. @...2F=i?..uf $\ldots\} \ldots=\{\ldots\} . .=z$. ..."b. $\sim \%\}=\ldots u 5$.

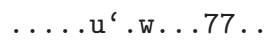
$\mathrm{H} \backslash \mathrm{y}$...u. . D. .V... $\mathrm{K}\} \mathrm{x} \ldots . . . .=. ?=. \mathrm{z}$ O.OG.D...J|A9GA I.....k..ct'u.i .\}.1...P.1.7.q. .\}....3o.l...w. $\ldots . \mathrm{H}\} \mathrm{r} \cdot \mathrm{j}) \ldots .$. d she was to get out again..

Fig. 13. 31-round SHA-0: second preimage (part 2) 